\title{
Transport of Disk-shaped Firebrands in a Turbulent Boundary Layer
}

\author{
KEISUKE HIMOTO and TAKEYOSHI TANAKA \\ Disaster Prevention Research Institute \\ Kyoto University \\ Uji, Kyoto 611-0011, Japan
}

\begin{abstract}
A transport model for a disk-shaped firebrand in 3D space has been formulated for the purpose of spotting simulation. In the model, firebrand transport is described by solving the conservation equations of momentum and angular-momentum, simultaneously. The airborne firebrand receives the aerodynamic forces and moment from the surrounding fluid, which varies in time and space due to the change in its location and orientation. An approximate form of the Navier-Stokes equations appropriate for low-Mach number flow was applied for the fluid motion. LES (Large Eddy Simulation) on buoyant flow downstream of a square heat source was carried out, and transport behaviors of various firebrands were investigated. Then, a scaling dimensionless parameter $B^{*}$ for the transport was derived. Numerically obtained mean travel distance to the windward direction, as well as its s.d. (standard deviation), were correlated with $B^{*}$ with reasonable accuracy.
\end{abstract}

KEYWORDS: firebrand, urban fires, wild fires, CFD, LES, scale model, modeling

\section{NOMENCLATURE}

$\begin{array}{llll}\text { Alphabets } & \text { Greeks } & \\ A_{P} & \text { Projected area }\left(\mathrm{m}^{2}\right) & \Delta & \text { Grid length scale }(\mathrm{m}) \\ B^{*} & \text { Dimensionless parameter }(-) & \theta & \text { Attack angle }(\mathrm{rad}) \\ c_{P} & \text { Specific heat }(\mathrm{kJ} /(\mathrm{kg} \cdot \mathrm{K})) & \mu & \text { Mean travel distance }(\mathrm{m}) \\ C & \text { Coefficient }(-) & \mu & \text { Viscosity }(\mathrm{kg} /(\mathrm{m} \cdot \mathrm{s})) \\ d_{P} & \text { Width }(\mathrm{m}) & \rho & \text { Density }\left(\mathrm{kg} / \mathrm{m}^{3}\right) \\ D & \text { Heat source length }(\mathrm{m}) & \sigma & \text { s.d. of travel distance }(\mathrm{m}) \\ F & \text { Force }\left(\mathrm{kg} \cdot \mathrm{m} / \mathrm{s}^{2}\right) & \boldsymbol{\omega}_{P} & \text { Firebrand spin }(\mathrm{rad} / \mathrm{s}) \\ g & \text { Acceleration of gravity }\left(\mathrm{m} / \mathrm{s}^{2}\right) & & \\ I_{P} & \text { Moment of inertia }\left(\mathrm{kg} \cdot \mathrm{m}^{2}\right) & \text { Subscripts } \\ k & \text { Conductivity }(\mathrm{kW} /(\mathrm{m} \cdot \mathrm{K})) & B & \text { Buoyancy } \\ l_{P} & \text { Side length }(\mathrm{m}) & D & \text { Drag } \\ M & \text { Moment }\left(\mathrm{kg} \cdot \mathrm{m}^{2} / \mathrm{s}^{2}\right) & L & \text { Lift } \\ p & \text { Pressure }\left(\mathrm{kg} \cdot \mathrm{m} / \mathrm{s}^{2}\right) & M & \text { Moment } \\ \dot{Q} & \text { Heat release rate }(\mathrm{kW}) & P & \text { Firebrand } \\ t & \text { Time }(s) & R & \text { Relative } \\ T & \text { Temperature }(\mathrm{K}) & \infty & \text { Ambient } \\ u & \text { Flow velocity }(\mathrm{m} / \mathrm{s}) & & \\ u_{P} & \text { Firebrand velocity }(\mathrm{m} / \mathrm{s}) & & \\ U_{\infty} & \text { Reference velocity }(\mathrm{m} / \mathrm{s}) & & \\ V & \text { Volume }\left(\mathrm{m}^{3}\right) & & \end{array}$




\section{INTRODUCTION}

Brand spotting is a dominant mechanism of fire-spread in urban/wild fires. Although the occurrence of such fires is not frequent, this will occasion considerable damage upon the built environment. In order to explore effective measures for reducing the loss, development of a rational model for firebrand transport is indispensable.

As transport of firebrands has received considerable attention, there is already number of models available. Tarifa, et al. [1], Lee, et al. [2], and Albini [3] assumed the brand as a minute or spherical particle, and provided methods for simulating its transport under the influence of drag and gravitational force as driving forces. Shiraishi et al. [4], and Huang et al. [5] have incorporated similar brand models into CFD (Computational Fluid Dynamics) simulations. However, in the event of a real fire, brand shape is not always ideal as it has been assumed in the past models. Rather, large portion of the observed firebrands in the past fires were non-spherical such as disk- or rod-shaped. Thus, the aerodynamic forces assumed in such models may differ to the ones of observed firebrands, as they generally vary kaleidoscopically along rotation due to the continuous change in their projected area. The work done by Woycheese, et al. [6], which simulated the transport of a disk in a 2D buoyant flow, is one of the few examples that have considered the shape-effect.

In this report, we modeled Lagrangean motion of a disk-shaped firebrand in a 3D buoyant flow. The aerodynamic forces and moment exerted upon the firebrand were calculated from the fluid motion obtained by solving an approximate form of the Navier-Stokes equations appropriate for low-Mach number flow. Using the model, LES (Large Eddy Simulation) on scattering of several firebrands was carried out, and their spotting behaviors were investigated.

\section{OUTLINE OF THE MODEL}

Schematic diagram of the model is shown in Fig. 1. Firebrand released above a heat source receives the drag and lift from the surrounding fluid, and the gravitational force to the vertical direction. It receives prevailing effect of the buoyant plume near the heat source and elevates its altitude, as well as it is transported to the downwind. Yet, when it is transported far from the heat source, it starts to decrease its altitude and finally drops on the ground, as the effect of gravity becomes dominant. During the transport, rotational moment is also exerted on the firebrand under the influence of fluid motion. This gives continuous change in the firebrand orientation, and correspondingly, the magnitude of exerted forces. The effect of combustion, which brings the change in mass and shape of the firebrand, is neglected in the current model.

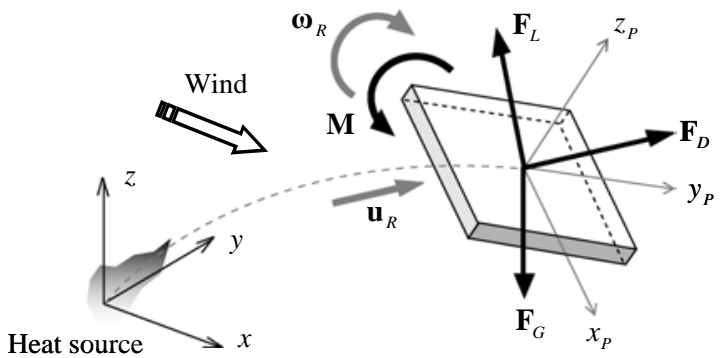

Fig. 1. Transport model for a disk-shaped firebrand driven by a buoyant flow. 
We described the motion of a firebrand with its velocity $\mathbf{u}_{P}$ and spin $\boldsymbol{\omega}_{P}$. These are defined with $x_{P} y_{P} z_{P}$-coordinate fixed on the brand. The origin is set to its center of gravity, $x_{P}$ and $y_{P}$ axes to be orthogonal to the edges, and $z_{P}$ axis to the direction of thickness. Thus, these axes form a principal-axes-of-inertia, and provide a simplified expression for the moment-of-inertia. An additional coordinate xyz whose center of gravity set to the heat source, is needed on the ground in order to trace the transport behaviors of the firebrand. Here, $x$ axis is set to be parallel to the wind direction, $z$ axis to the vertical direction, $y$ axis to be perpendicular to the other two axes.

\section{FIREBRAND MOTION}

\section{Equations of Motion}

In the model, brand is assumed as a rigid object. When the change in mass and shape are neglected, the advection and rotation of the brand are described with the following conservation equations of kinetic-momentum and angular-momentum, respectively,

$\rho_{P} V_{P} \frac{d \mathbf{u}_{P}}{d t}=\mathbf{F}_{D}+\mathbf{F}_{L}+\mathbf{F}_{G}$

$\frac{d}{d t}\left(\mathbf{I}_{P} \cdot \boldsymbol{\omega}_{P}\right)=\mathbf{M}$,

where $\rho_{P}$ is the density, $V_{P}$ is the volume, $\mathbf{F}_{D}$ is the drag, $\mathbf{F}_{L}$ is the lift, $\mathbf{F}_{G}$ is the gravitational force, $\mathbf{I}_{P}$ is the moment-of-inertia, $\mathbf{M}$ is the moment. As $x_{P}, y_{P}$, and $z_{P}$ axes are set so as to form a principal-axes-of-inertia, the tensor $\mathbf{I}_{P}$ is given by,

$$
\mathbf{I}_{P}=\frac{\rho_{P} V_{P}}{12}\left(\begin{array}{ccc}
l_{P, y}^{2}+l_{P, z}^{2} & 0 & 0 \\
0 & l_{P, x}^{2}+l_{P, z}^{2} & 0 \\
0 & 0 & l_{P, x}^{2}+l_{P, y}^{2}
\end{array}\right)
$$

where $l_{P, x}, l_{P, y}$, and $l_{P, z}$ are the side length of each axes. The velocity $\mathbf{u}_{P}$ and spin $\boldsymbol{\omega}_{P}$ are calculated in a time series by solving Eqs. 1, and 2, simultaneously. Detailed numerical procedure is outlined in the previous report [7].

\section{Forces and Moment}

Drag force $\mathbf{F}_{D}$ drives the brand to the direction of the relative velocity $\mathbf{u}_{R}$, and lift force $\mathbf{F}_{L}$ to the direction of the vector product $\mathbf{u}_{R} \times \boldsymbol{\omega}_{R}$. The magnitudes of these aerodynamic forces are both proportional to the kinetic energy,

$$
\mathbf{F}_{D}=C_{D} A_{P} \cdot \frac{1}{2} \rho\left|\mathbf{u}_{R}\right| \mathbf{u}_{R},
$$


$\mathbf{F}_{L}=C_{L} A_{P} \cdot \frac{1}{2} \rho\left|\mathbf{u}_{R}\right|^{2} \frac{\mathbf{u}_{R} \times \boldsymbol{\omega}_{R}}{\left|\mathbf{u}_{R} \times \boldsymbol{\omega}_{R}\right|}$.

Note that $C_{D}$ and $C_{L}$ are the coefficients of drag and lift, respectively. $\rho$ is the fluid density, and $A_{P}$ is the projected area. The gravitational force $\mathbf{F}_{G}$, which is exerted to the vertical direction, is given as,

$\mathbf{F}_{G}=\left(\rho_{P}-\rho\right) V_{P} \mathbf{g}$,

where $\mathbf{g}$ is the acceleration due to gravity. The moment $\mathbf{M}$ acts to the direction that reduces the relative spin $\boldsymbol{\omega}_{R}$, which is expressed with the coefficient $C_{M}$ as,

$\mathbf{M}=-C_{M} A_{P} l_{P} \cdot \frac{1}{2} \rho\left|\mathbf{u}_{R}\right|^{2} \frac{\boldsymbol{\omega}_{R}}{\left|\boldsymbol{\omega}_{R}\right|}$.

In order to calculate the above forces and moment, the aerodynamic coefficients $C_{D}, C_{L}$, and $C_{M}$ need to be modeled additionally. It is generally acknowledged that these are expressed as functions of aerodynamic parameters such as Reynolds number. Yet, as there is little information available, we tentatively describe them from the existing experimental results [8] carried out at the Reynolds number of 40000, as follows (Fig. 2),

$C_{D}=1.1|\sin \theta|$,

$C_{L}=\min \{2.0|\sin \theta|, 1.2-3.0(|\sin \theta|-0.6)\}$,

$C_{M}=\min \{0.35|\sin \alpha|, 0.14-2.3(|\sin \alpha|-0.4)\}$.

where $\theta$ is the attack angle of the relative velocity.

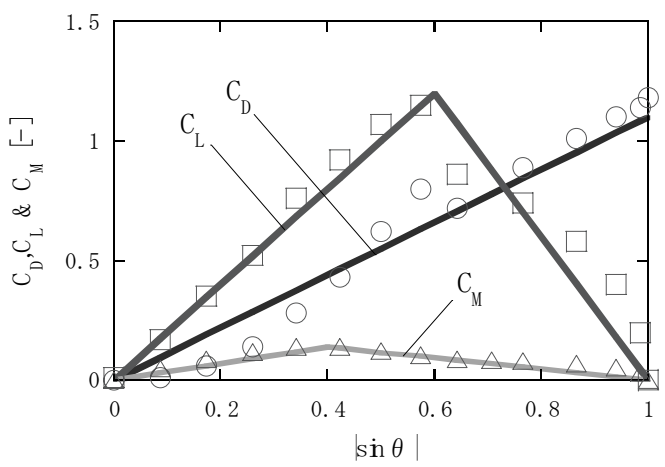

Fig. 2. Aerodynamic coefficients.

\section{FLUID MOTION}

An approximate form of the Navier-Stokes equations [9] is used for LES of fluid motion. Neglecting the effect of firebrand motion on fluid motion, the filtered continuity, momentum, enthalpy, and state equations are expressed as, 


$$
\begin{aligned}
& \frac{\partial \bar{\rho}}{\partial t}+\frac{\partial \overline{\rho u}_{i}}{\partial x_{i}}=0, \\
& \frac{\partial \overline{\rho u}_{i}}{\partial t}+\frac{\partial \overline{\rho u}_{i} \tilde{u}_{j}}{\partial x_{i}}=-\frac{\partial \bar{p}}{\partial x_{i}}+\frac{\partial}{\partial x_{j}}\left(2 \mu \tilde{S}_{i j}\right)-\left(\bar{\rho}-\rho_{\infty}\right) g \delta_{i 3}-\frac{\partial \tau_{i j}}{\partial x_{j}}, \\
& \frac{\partial \overline{\rho T}}{\partial t}+\frac{\partial \bar{\rho} \tilde{u}_{j} \tilde{T}}{\partial x_{j}}=\frac{\partial}{\partial x_{j}}\left(\frac{k}{c_{P}} \frac{\partial \tilde{T}}{\partial x_{j}}\right)+\frac{\overline{\dot{q}^{\prime \prime \prime}}}{c_{P}}-\frac{\partial h_{j}}{\partial x_{j}}, \\
& p_{\infty}=\bar{\rho} R \tilde{T}
\end{aligned}
$$

where, $\rho$ is the density, $u$ is the velocity, $p_{\infty}$ is the mean ambient pressure, $p^{\prime}$ is the pressure perturbation, $T$ is the temperature, $\mu$ is the viscosity, $k$ is the thermal conductivity, $c_{P}$ is the specific heat, $\dot{q}^{\prime \prime \prime}$ is the heat release rate per unit volume, and $R$ is the gas constant, and $\tilde{S}_{i j}=\left(\partial \tilde{u}_{i} / \partial x_{j}+\partial \tilde{u}_{j} / \partial x_{i}\right) / 2$ is the strain rate tensor. The symbols with (-) and ( $\sim$ ) designate volume filtered values, and Favre averaged values, respectively.

The equations presented above also require models for the SGS (sub grid scale) stress $\tau_{i j}$ and SGS heat flux $h_{j}$. These are parameterized by eddy viscosity models as follow,

$$
\begin{aligned}
& \tau_{i j}=\bar{\rho}\left(\widetilde{u_{i} u_{j}}-\tilde{u}_{i} \tilde{u}_{j}\right)=\frac{1}{3} \delta_{i j} \tau_{k k}-2 \bar{\rho}\left(C_{S} \Delta\right)^{2}\left|\tilde{S}_{i j}\right|\left(\tilde{S}_{i j}-\frac{1}{3} \tilde{S}_{k k} \delta_{i j}\right), \\
& h_{j}=\bar{\rho} c_{P}\left(\widetilde{u_{j} T}-\tilde{u}_{j} \tilde{T}\right)=-\bar{\rho} c_{P} \frac{\left(C_{S} \Delta\right)^{2}\left|\tilde{S}_{i j}\right|}{\operatorname{Pr}} \frac{\partial \tilde{T}}{\partial x_{j}},
\end{aligned}
$$

where $C_{S}$ is the Smagorinsky constant, $\operatorname{Pr}$ is the turbulent Prandtl number, $\Delta$ is the grid length scale, and $\left|\tilde{S}_{i j}\right|$ is the magnitude of the strain tensor.

\section{SCATTERING OF FIREBRANDS IN A TURBULENT BOUNDARY LAYER}

Fires involving brand spotting, such as urban fires or wild fires, generally take place in a turbulent boundary layer, in which building structures and vegetations play as the turbulence generating roughness. Here, we assumed a heat source in such an environment, and discharged several types of firebrands into the flow field.

The assumed flow conditions are summarized in Tables 1 and 2. As the combustion of fuel is not considered in the current model, heat release rate $\dot{Q}$ was uniformly distributed upon the first cells above the ground. Time-dependent turbulent flow [10] was generated in advance of the numerical simulation, and applied as the inflow boundary condition.

The side length $l_{P}$, thickness $d_{P}$, and density $\rho_{P}$ of the firebrands are varied as shown in Table 3. They were discharged at the height of $D$, above the leeward edge of the heat 
source on the ground. Initial velocity and spin of the brand were set equal to the instantaneous fluid velocity and spin of the relevant position, respectively. Yet, Initial orientation of the firebrand was given at random.

Table 1. Flow conditions.

\begin{tabular}{l|l}
\hline \hline Heat source size & $\mathrm{D}=0.08 \mathrm{~m}\left(\mathrm{~A}=0.064 \mathrm{~m}^{2}\right)$ \\
\hline Heat release rate & $\mathrm{Q}=4.0 \mathrm{~kW}$ \\
\hline Mean inflow profile & $U_{\infty}=5.0 \mathrm{~m} / \mathrm{s}, u(z) / U_{\infty}=\left\{\begin{array}{c}\left(z / z_{\infty}\right)^{1 / 4}\left(z \leq z_{\infty}\right) \\
1 \quad\left(z \geq z_{\infty}\right)\end{array}\right.$ \\
\hline Computational domain & $31 \mathrm{D}(x) \times 12.5 \mathrm{D}(y) \times 12.5 \mathrm{D}(z)$ \\
\hline Number of cells & $124(x) \times 50(y) \times 50(z)$ \\
\hline Time step & $\Delta t \cdot U_{\infty} / D=6.25 \times 10^{-3}(-)$ \\
\hline \hline
\end{tabular}

Table 2. Boundary conditions.

\begin{tabular}{l|l}
\hline \hline Inflow & Time-dependent turbulent inflow generated in advance [10] \\
\hline Outflow & Convective outflow condition \\
\hline Side \& top & Free-slip condition \\
\hline bottom & Velocity: no-slip condition, Temperature: free-slip condition \\
\hline \hline
\end{tabular}

Table 3. Firebrand conditions.

\begin{tabular}{|c|c|c|c|c|c|}
\hline & & $l_{P} / D(-)$ & $d_{P} / D(-)$ & $l_{P} / d_{P}(-)$ & $\rho_{P}\left(\mathrm{~kg} / \mathrm{m}^{3}\right)$ \\
\hline \multirow{3}{*}{ A } & 1 & \multirow{3}{*}{$1 / 200$} & \multirow{3}{*}{$1 / 2000$} & \multirow{3}{*}{10} & 50.0 \\
\hline & 2 & & & & 100.0 \\
\hline & 3 & & & & 150.0 \\
\hline \multirow{3}{*}{ B } & 1 & \multirow{9}{*}{$1 / 100$} & \multirow{3}{*}{$1 / 1250$} & \multirow{3}{*}{12.5} & 50.0 \\
\hline & 2 & & & & 100.0 \\
\hline & 3 & & & & 150.0 \\
\hline \multirow{3}{*}{$\mathrm{C}$} & 1 & & \multirow{3}{*}{$1 / 1500$} & \multirow{3}{*}{15} & 50.0 \\
\hline & 2 & & & & 100.0 \\
\hline & 3 & & & & 150.0 \\
\hline \multirow{3}{*}{$\mathrm{D}$} & 1 & & \multirow{3}{*}{$1 / 1000$} & \multirow{6}{*}{10} & 50.0 \\
\hline & 2 & & & & 100.0 \\
\hline & 3 & & & & 150.0 \\
\hline \multirow{3}{*}{$\mathrm{E}$} & 1 & \multirow{3}{*}{$3 / 200$} & \multirow{3}{*}{$3 / 2000$} & & 50.0 \\
\hline & 2 & & & & 100.0 \\
\hline & 3 & & & & 150.0 \\
\hline
\end{tabular}

\section{Behaviors of Brand Scattering}

Examples of the trajectories and the ground-level distribution of the released brands for different density conditions are shown in Fig. 3a-c. Released brands were transported to the downwind with the buoyancy-induced flow behind the heat source. Most of the brands fell on the ground soon after the discharge. Yet, some brands were transported as far as the boundary of the computational domain. 
The obtained peaks of the travel distance to the wind direction were about $7 D$ for $\rho_{P}=50.0 \mathrm{~kg} / \mathrm{m}^{3}, 5 D$ for $\rho_{P}=100.0 \mathrm{~kg} / \mathrm{m}^{3}$, and $3 D$ for $\rho_{P}=150.0 \mathrm{~kg} / \mathrm{m}^{3}$, i.e., smaller the density, larger the travel distance. It is readily perceived that the firebrand was transported further as it easily followed the buoyancy-induced upward current when its inertia was small. The gravitational force is the another factor which influenced the travel distance, as it is almost proportional to density as shown in Eq. 6. On the contrary, there was no apparent difference in the scattering range to the direction perpendicular to the mainstream.

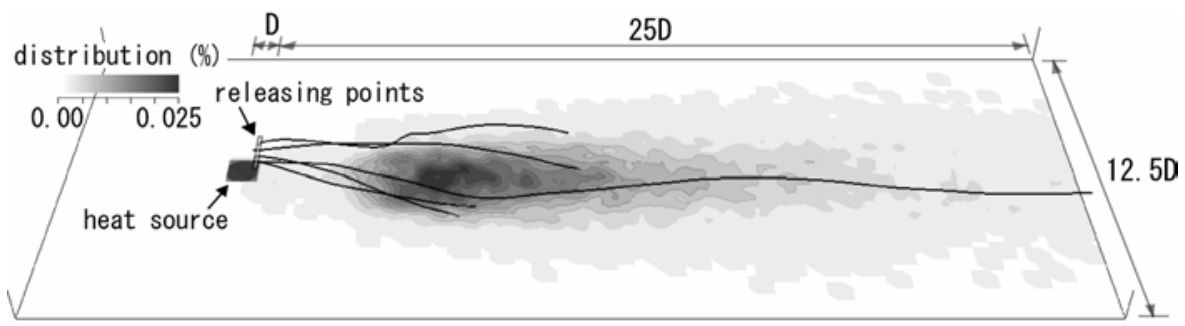

Fig. 3a. Distribution of fallen firebrands (D1: $\rho_{P}=50.0 \mathrm{~kg} / \mathrm{m}^{3}$ ).

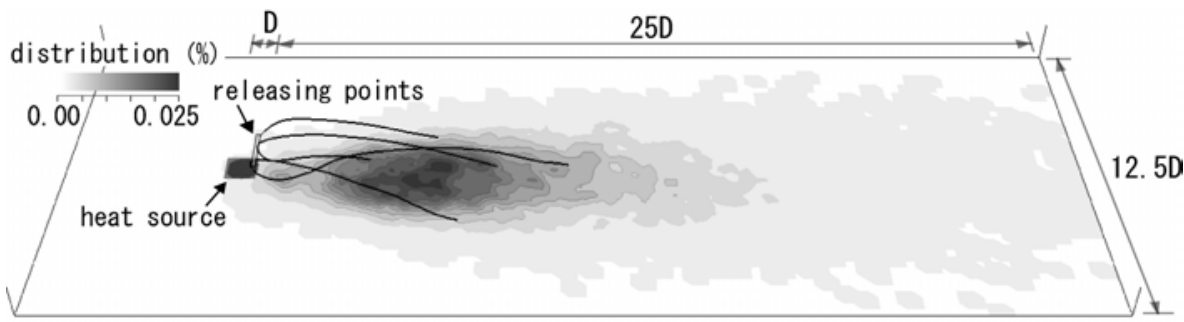

Fig. 3b. Distribution of fallen firebrands (D2: $\rho_{P}=100.0 \mathrm{~kg} / \mathrm{m}^{3}$ ).

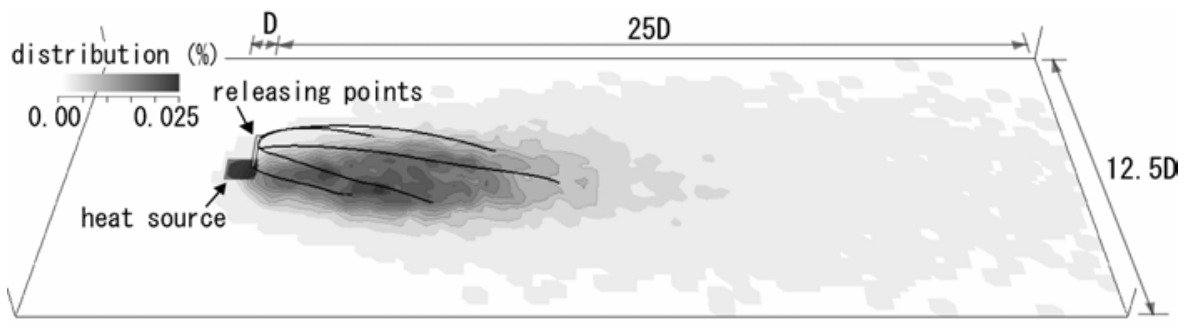

Fig. 3c. Distribution of fallen firebrands (D1: $\rho_{P}=150.0 \mathrm{~kg} / \mathrm{m}^{3}$ ).

\section{SCALE MODEL FOR THE TRANSPORT}

\section{Dimensionless Parameter}

In order to generalize the numerical results, we now derive a scaling parameter for the travel distance. The general trajectory of a firebrand is described in Fig. 4. Once released, firebrand is uplifted by the strong buoyant flow, and transported to the downwind with the airflow over the area. As the brand gets further from the fire, the gravity effect prevails the buoyancy effect. Thus, the brand starts to decrease its altitude and finally falls upon the ground. Although, such behavior is a motion in three dimensions by nature, 
we treat it as a two dimensional motion of predominant wind-direction $x$, and vertical direction $z$, for simplicity.

At first, assuming that the wind effect is strong, and the firebrand follows the current motion. Then, the motion to the $x$-direction is described as,

$u_{P}=U_{\infty}$.

Whereas to the $z$-direction, the equation of motion is described with the buoyancy force $F_{B}$ to the upward, and gravitational force $F_{G}$ to the downward, as follows,

$\rho_{P} V_{P} \frac{d w_{P}}{d t}=F_{B}-F_{G}$,

in which $F_{B}$ is a resultant force of the drag and lift. Approximating the upward velocity of a plume in cross wind to the one in a quiescent ambient condition [11], $F_{B}$ is given by,

$$
F_{B} \cong C A_{P} \cdot \frac{1}{2} \rho_{\infty} W_{\infty}^{2} \cong C_{B} A_{P} \cdot \frac{1}{2} \rho_{\infty} g z\left(\frac{\dot{Q}}{\rho_{\infty} C_{P} T_{\infty} g^{1 / 2} z^{5 / 2}}\right)^{2 / 3} .
$$

As the density of firebrand is generally large compared to the one of the surrounding air, gravitational force in Eq. 6 is approximated as,

$F_{G} \cong \rho_{P} V_{P} g$.

When a firebrand reaches at its highest altitude $z_{P}$, the acceleration of the vertical direction becomes almost zero in Eq. 18. So, with Eqs. 19 and 20, $z_{P}$ is given by,

$z_{P} \cong\left(\frac{\dot{Q}}{\rho_{\infty} c_{P} T_{\infty} g^{1 / 2} d_{P}^{5 / 2}}\right)\left(\frac{\rho_{\infty}}{\rho_{P}}\right) \cdot d_{P}$.

From then on, the gravitational force prevails the buoyancy force $\left(F_{B}<<F_{G}\right)$, and the acceleration of the vertical direction approaches to the one due to gravity $g$. As the brand will be accelerated uniformly in such a condition, the highest altitude $z_{P}$ can be described with the time to reach the ground $t_{P}^{\prime}$ as,

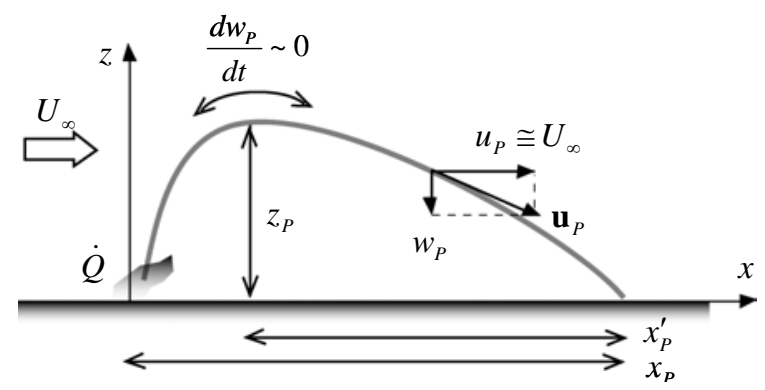

Fig.4. Travel distance of a firebrand. 
$z_{P} \cong \frac{1}{2} g t_{P}^{\prime 2}$

Solving the above Eqs. 21 and 22, $t_{P}^{\prime}$ is obtained as,

$t_{P}^{\prime} \cong\left(\frac{\rho_{P}}{\rho_{\infty}}\right)^{-3 / 4}\left(\frac{\dot{Q}}{\rho_{\infty} c_{P} T_{\infty} g^{1 / 2} d_{P}^{5 / 2}}\right)^{1 / 2}\left(\frac{2 d_{P}}{g}\right)^{1 / 2}$.

So, with Eq. 17, the travel distance to the $x$-direction is described as,

$$
x_{P}^{\prime} \cong U_{\infty} t_{P}^{\prime}=\sqrt{2}\left\{\frac{U_{\infty}}{\left(g d_{P}\right)^{1 / 2}}\left(\frac{\rho_{P}}{\rho_{\infty}}\right)^{-3 / 4}\left(\frac{\dot{Q}}{\rho_{\infty} c_{P} T_{\infty} g^{1 / 2} d_{P}^{5 / 2}}\right)^{1 / 2}\right\} \cdot d_{P} .
$$

Yet, as $x_{P}^{\prime}$ is only a stream-wise travel distance after the brand has reached at its highest altitude, $x_{P}^{\prime}$ does not correspond to the distance from the heat source $x_{P}$. However, as $x_{P}^{\prime}$ occupies not a few portion of overall travel distance $x_{P}$, a certain sort of relation is expected between these two parameters, i.e.,

$x_{P}=f\left(x_{P}^{\prime}\right)$.

Here, we normalize the right hand side of Eq. 24 with the characteristic length scale $D$, and define a dimensionless parameter,

$$
B^{*} \equiv \frac{U_{\infty}}{(g D)^{1 / 2}}\left(\frac{\rho_{P}}{\rho_{\infty}}\right)^{-3 / 4}\left(\frac{d_{P}}{D}\right)^{-3 / 4}\left(\frac{\dot{Q}}{\rho_{\infty} c_{P} T_{\infty} g^{1 / 2} D^{5 / 2}}\right)^{1 / 2} .
$$

Thus, with Eq. 25, $x_{P}$ is formulated as follows,

$\frac{X_{P}}{D}=f\left(B^{*}\right)$.

Incidentally, the width $d_{P}$ is the sole parameter related to the firebrand shape, which is involved in the derived dimensionless parameter $B^{*}$.

\section{Verification of The Scale Model}

The travel distances to the $x$ - and $y$-directions (mean and s.d. (standard deviation)) are plotted against the derived parameter $B^{*}$ in Figs. 5 and 6, respectively. These plots are well aligned on log-log graph and can be approximated with the following expressions,

$$
\left.\left.\begin{array}{r}
\mu_{x} / D=0.47 B^{* 2 / 3} \\
\sigma_{x} / D=0.88 B^{* 1 / 3}
\end{array}\right\} \text {, and } \begin{array}{r}
\mu_{y} / D=0.00 \\
\sigma_{y} / D=0.92
\end{array}\right\} \text {. }
$$


Although the results for the $x$-direction $\left(\mu_{x}\right.$ and $\sigma_{x}$ ) were well correlated with $B^{*}$, the results for the $y$-direction ( $\mu_{y}$ and $\sigma_{y}$ ) were shown to be independent of $B^{*}$. This implies that the brand shape has no apparent effect on the transport behaviors to the $y$ direction. However, this conclusion relies on the results of a sole flow field condition, and a different conclusion may be obtained with additional numerical simulation.

Regarding the distribution of fallen firebrands on the ground, the scattering distribution to the $x$-direction is now approximated with the log-normal function as follows,

$$
p(x)=\frac{1}{\sqrt{2 \pi} \sigma_{L, x} x} \exp \left\{-\frac{\left(\ln x-\mu_{L, x}\right)^{2}}{2 \sigma_{L, x}^{2}}\right\} \quad(0<x<\infty)
$$

in which, $\mu_{L, x}$ and $\sigma_{L, x}$ are the mean and s.d. values of $\ln (x)$. These are calculated with $\mu_{x}$ and $\sigma_{x}$ as follows,

$\mu_{L, x}=\ln \frac{\mu_{x}}{\sqrt{1+\left(\sigma_{x} / \mu_{x}\right)^{2}}}$, and $\sigma_{L, x}=\sqrt{\ln \left\{1+\left(\sigma_{x} / \mu_{x}\right)^{2}\right\}}$.

While for the $y$-direction, the distribution is approximated with the normal function,
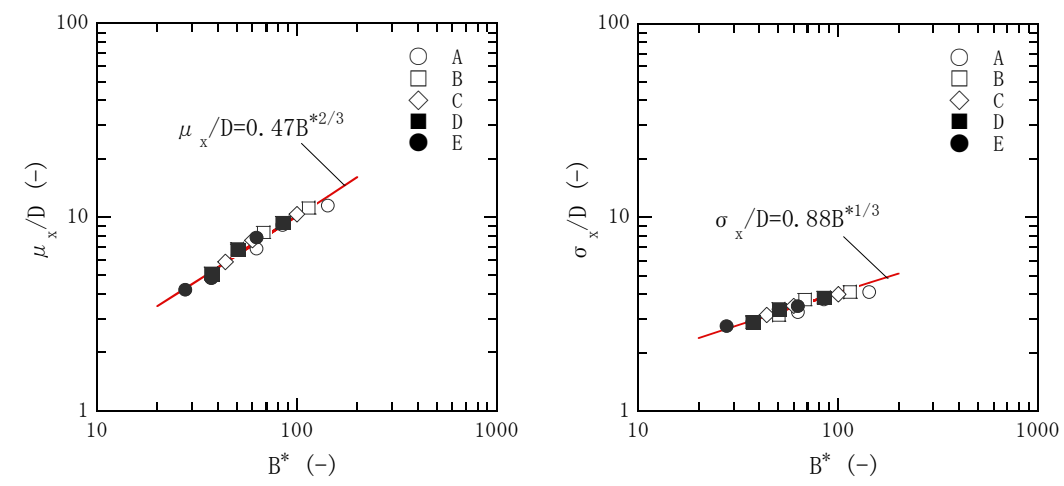

Fig. 5. Travel distance to $x$-direction (mean and s.d.).
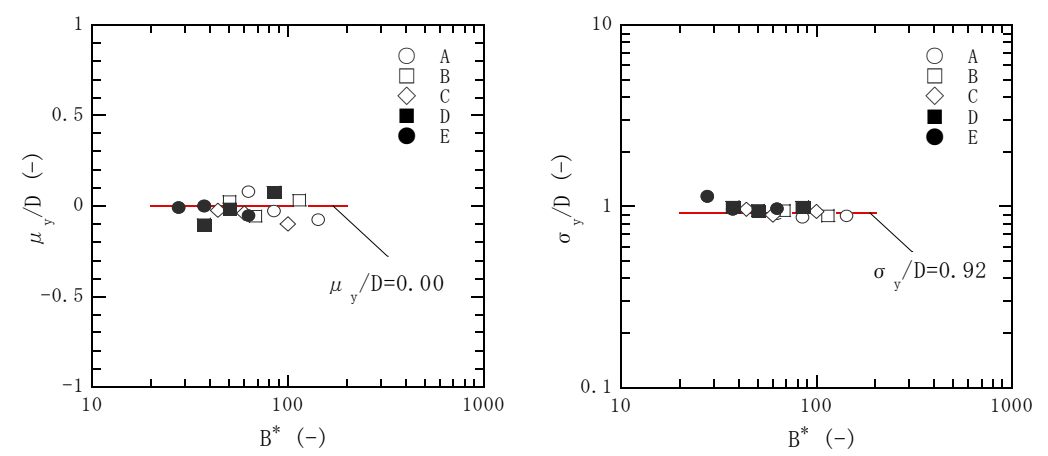

Fig. 6. Travel distance to $y$-direction (mean and s.d.). 
$q(y)=\frac{1}{\sqrt{2 \pi} \sigma_{y}} \exp \left\{-\frac{\left(y-\mu_{y}\right)^{2}}{2 \sigma_{y}^{2}}\right\} \quad(-\infty<y<\infty)$.

Estimated scattered distributions by Eqs. 29 and 31 are compared with the LES data for the cases D1, D2, and D3, as shown in Fig.7a-c. The overall agreements are reasonably well. Yet, as a log-normal function was adopted as the approximate function, kurtosis was overestimated for the case shown in Fig.7c, at which the fallen brands gathered near the discharged point.
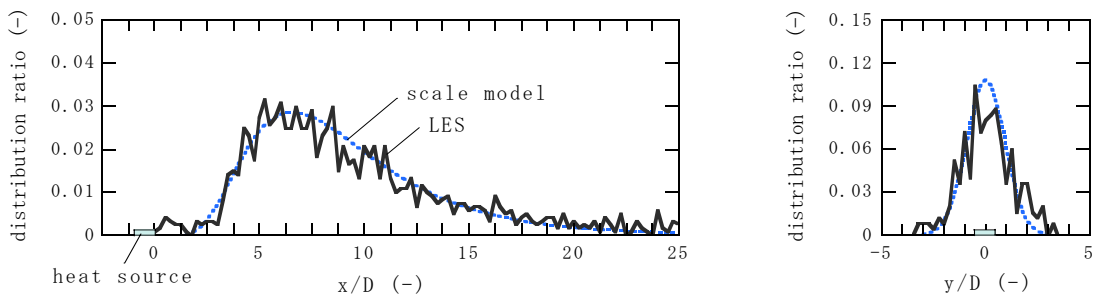

Fig. 7a. Distribution of fallen firebrands (D1: $\rho_{P}=50.0 \mathrm{~kg} / \mathrm{m}^{3}$ ).
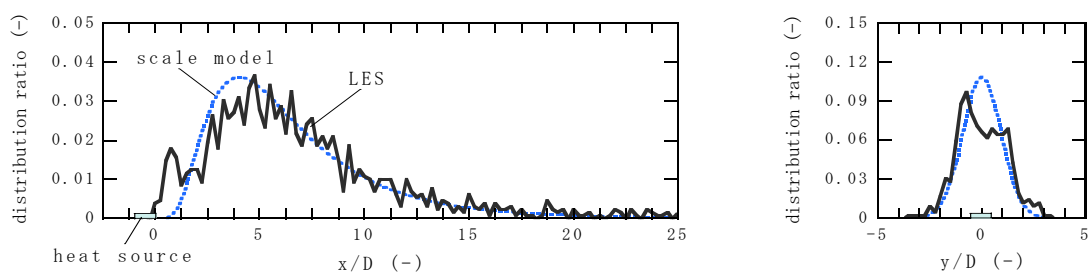

Fig. 7b. Distribution of fallen firebrands (D2: $\rho_{P}=100.0 \mathrm{~kg} / \mathrm{m}^{3}$ ).
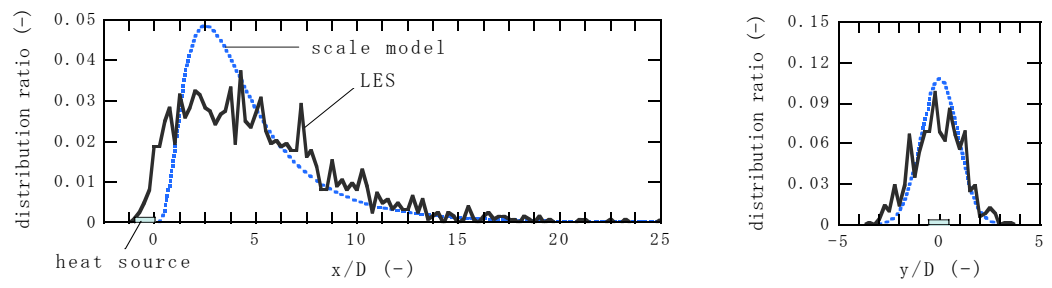

Fig. 7c. Distribution of fallen firebrands (D3: $\rho_{P}=150.0 \mathrm{~kg} / \mathrm{m}^{3}$ ).

\section{CONCLUDING REMARKS}

Transport model for a disk-shaped firebrand has been formulated. With the model, LES on brand scattering behind a heat source were carried out in a turbulent boundary layer, and the effect of some brand properties were investigated. The obtained numerical results were generalized with a newly derived dimensionless parameter $B^{*}$ reasonably well.

However, as some important phenomena were not regarded in the current model, the obtained results are tentative. Changes in mass, shape, or aerodynamic coefficients due to 
the brand combustion are ones of the examples. These are the topics which will need further considerations in the future.

\section{REFERENCES}

[1] Tarifa, C.S., del Notario, P.P. and Moreno, F.G., "On The Flight Paths and Lifetimes of Burning Particles of Wood,” 10th Symp. (Int.) Comb., pp. 10211037, 1965.

[2] Lee, S.L. and Hellman, J.M., "Firebrand Trajectory Study Using an Empirical Velocity-dependent Burning Law,” Comb. Flame, Vol. 15, pp. 265-274, 1970.

[3] Albini, F.A., "Transport of Firebrands by Line Thermals," Comb. Sci. Tech., Vol. 32, pp. 277-288, 1983.

[4] Shiraishi, Y., Kato, S., Yoshida, S., and Murakami, S., "Numerical Analysis of Scatter of Fire Brand within Urban Fire Spread," J. Architectural Plan. and Environ. Eng., No. 546, pp.187-192, 2001 (in Japanese).

[5] Huang, H., Ooka, R., Kato, S., Hayashi, Y., and Ootake, H., "CFD Analysis on Combustion Modeling and Firebrand Scattering in Urban Fire," Proc. 18th Symp. Numerical Simulation of Turbulence, pp. 7-12, 2003, (in Japanese).

[6] Woycheese, J.P. and Pagni, P.J., "Combustion Models of Wooden Brands," Proc., 3rd Int. Conf. Fire Res. Eng., pp. 53-71, 1999.

[7] Himoto, K., Maruyama, T. and Tanaka, T., “A Study on The Brand Spotting in Urban Fire," Proc. 10 ${ }^{\text {th }}$ INTERFLAM, Vol. 2, pp. 1039-1050, 2004.

[8] Tachikawa, M. and Fukuyama, M., "Trajectories and Velocities of Typhoongenerated Missiles,” Transaction of Architectural Institute of Japan, No. 302, pp. 1-11, 1981 (in Japanese).

[9] Rehm, R. and Baum, H., "The Equations of Motion for Thermally Driven, Buoyant Flows," J. Res. National Bureau of Standards, Vol. 83, No. 3, pp. 297308, 1978.

[10] Kataoka, H. and Mizuno, M., "Numerical Flow Computation Around 3D Square Cylinder Using Inflow Turbulence," Transaction of Architectural Institute of Japan, No. 523, pp. 71-77, 1999, (in Japanese).

[11] Morton, B.R., Taylor, G. and Turner, J.S., "Turbulent Gravitational Convection from Maintained and Instantaneous Sources,” Proc., Royal Soc., Vol. 234A, pp. 1-23, 1956. 